\title{
Secure and Efficient Routing Protocol (E-ARAN) for Ad-Hoc Network
}

\author{
M.Gurunadhababu, G.Archanadevi, Mahendra P. Sharma
}

\begin{abstract}
The working scope of Ad-hoc system will spread in coming future because of dynamic nature. Be that as it may, there will be the danger of spreading incorrectly directing data, bundle dropping and particular sending in the system which further prompts exceptional sort of assaults [1]. Existing validated directing conventions for Ad-hoc system neglects to identify and guard against such sort of assaults in the portable impromptu system. In this manner, if pernicious hub hack the bundles and make the changes, deliberately drop control or information parcels, the present determination of existing steering conventions can't identify or protect against such verified egotistical nodes. This shortcoming in ARAN detail will bring about the aggravation of the impromptu system and the misuse of the system data transfer capacity. In this examination paper, an answer is proposed to represent this sort of assaults.
\end{abstract}

Key-words: AHN-Ad-hoc arrange, ARAN-Authenticated Routing Protocol for Ad-hoc Network, TTP-Trusted Third Party, RDP-Route Discovery Packet, REP-Route Reply Packets

\section{INTRODUCTION}

Conceptual Execution assessment of TCP net web page on line net web site visitors in OBS systems has been under raised exam, whilst you recollect that TCP develops most of internet net website online traffic. As a sturdy and straightforwardly open test shape, ns2 has been generally applied for thinking about TCP/IP systems; anyhow ns2 desires a remarkable package deal of the elements for reflecting optical burst looking for and advancing systems. on this paper, a ns2 basically based totally OBS reenactment framework (nOBS), it's miles toiled for dissecting burst gathering, reserving and hassle goals includes in OBS structures is set up. The middle detail and association request in OBS are connected in nOBS for making optical places of work and optical corporations. The path, acknowledgment and departure reputation factor functionalities are joined at once into an regular optical center attitude structuring, which consolidates executives liable for burstification, dealing with and masterminding. The consequences of burstification parameters, e.G., burstification demolish, burst term and quantity of burstification assist beautify popularity, on TCP execution are researched the use of nOBS for great TCP translations and simple shape topologies. The project plan of libraries we made for this element is called Multi InteRfAce flow into Layer Extension for ns2 (MIRACLE). They supplant

\footnotetext{
Revised Manuscript Received on September 10, 2019.

Dr.M.Gurunadhababu, Professor, CMR Institute of Technology, Hyderabad, Telangana, India

(E-mail: mgurunadhababu@gmail.com)

Mrs.G.Archanadevi, Assistant Professor, CMR Institute of Technology, Hyderabad, Telangana, India

(E-mail: archana0286@gmail.com)

Mahendra P. Sharma, Assistant Professor- IIMT College of Engg,Gr.Noida, U.P, India
}

the functionalities provided via manner of the machine Simulator ns2 thru giving a gainful and set up motor for searching after flow into-layer messages and, at the indistinguishable time, empowering the combination of different modules inner each layer of the presentation stack. for instance, diverse shape, association, MAC or actual layers can be settled and applied internal a practically equal cognizance. The repercussions of this are mind boggling. As an difficulty of first essentialness, the shape engages the execution and the reenactment of front line correspondence systems in ns2. moreover, due to its organized noteworthy, the code might be littler, re-usable and extensible.

\section{II.TUNNELING}

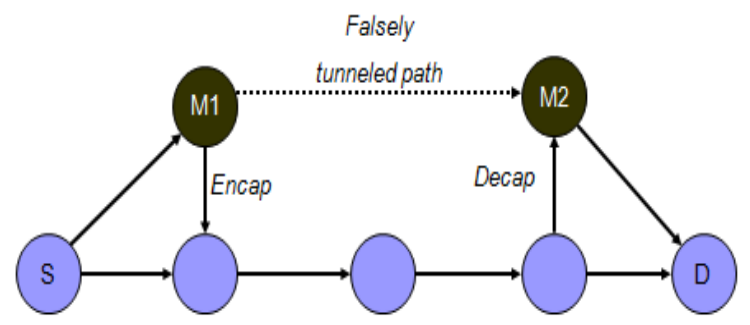

Fig: 1 Tunneling

at the factor on the identical time as M1 gets a RDP from S, M1 epitomizes the RDP and entries it to M2 through a slicing element certainties direction, for this situation M1$>\mathrm{A}->\mathrm{B}->\mathrm{C}->\mathrm{M} 2$.on the equal time as $\mathrm{M} 2$ receives the exemplified RDP, and it propels the RDP at once to $\mathrm{D}$ as notwithstanding the reality that it had honestly voyage $\mathrm{S}->$ M1 - > M2 - > D. Neither M1 nor M2 supplant the bundle deal header to mirror that the RDP similarly dared to every piece of the manner A->B->C. After direction disclosure, it appears to the reason that there are guides from $\mathrm{S}$ of conflicting period: $\mathrm{S}->\mathrm{A}->\mathrm{B}->\mathrm{C}->\mathrm{D}$ and $\mathrm{S}->\mathrm{M} 1->\mathrm{M} 2->\mathrm{D}$. at the off peril that M2 tunnels the RREP decrease lower back to M1, S must deceptively hold in mind the remarkable method to D via M1 a propelled selection (as a ways as route term) than the amazing approach to $\mathrm{D}$ with the guide of A. In our assumption, center detail A wants to get a bearing to middle $\mathrm{D}$.

\section{UNEVEN CRYPTOGRAPHIC SOLUTIONS}

indicates that use disproportionate cryptography to verify coordinating in portable particularly delegated systems require the nearness of a generally depended on in untouchable (TTP) 


\section{ARAN}

ARAN or confirmed controlling display recognizes and verifies in the direction of threatening video games with the guide of outcast and friends in explicitly named contraption. real ranges of ARAN encompass of a basis insistence approach favored with the aid of manner of a direction launch manner that guarantees thru and through certification. ARAN makes use of cryptographic articulation to perform its employer.

\section{(a) course Initiation Step:}

\section{set up 1}

every center issue, earlier than attempting to connect to the explicitly delegated framework, want to touch the confirmation grasp and sales a validation for its vicinity and open key

$\mathrm{A}:$ cert $\mathrm{A}=[\mathrm{IPA}, \mathrm{KA}+, \mathrm{t}, \mathrm{e}] \mathrm{KTT}$

affirmation contains of the IP control of An (IPA), the open key of $\mathrm{A}(\mathrm{KA}+)$, a timestamp ok of at the same time because the assist become made, and a length e at which the announcement ends. those variables are associated and stamped thru using KT-. The display widely known that each middle is aware of from the sooner the open key of the attestation hold close.

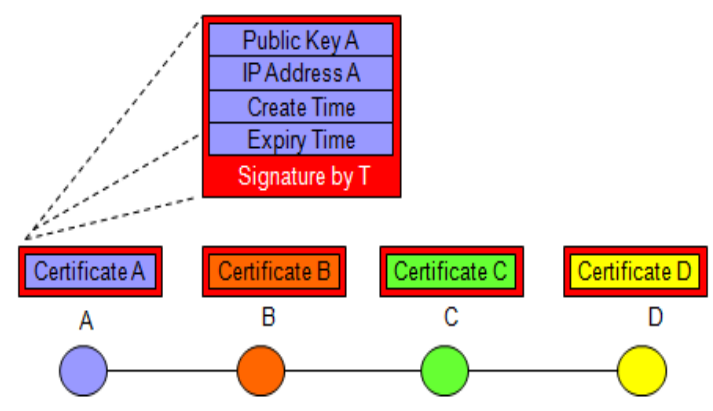

Trusted certificate server T

Fig 2: ARAN - initial Setup

From the start every center aspect has its very own genuinely considered one of a type affirmation made via way of relied on in validation server T.each middle in like way has a duplicate of T's open key, with a purpose to check severa helps.

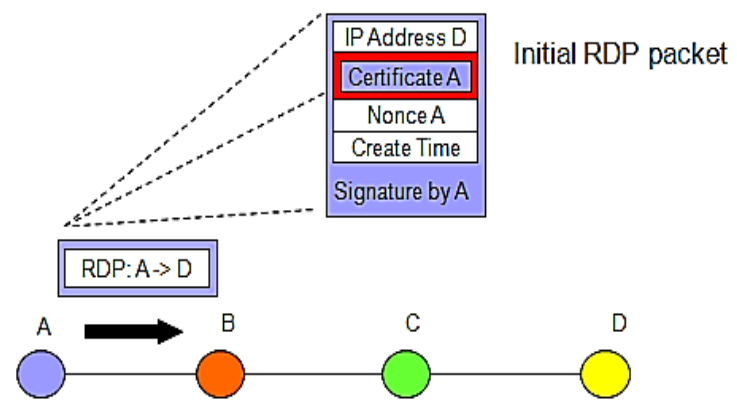

Fig three: ARAN - path Discovery

center thing A makes a RDP name for bundle for middle factor D. Middle point Its very private incorporates affirmation, and a quick time later signs and symptoms and symptoms the RDP package with its personal key. Middle element An at that element imparts this p.C. To its buddies. It seems that each neighbor can check the bundle in reality commenced from middle point A.

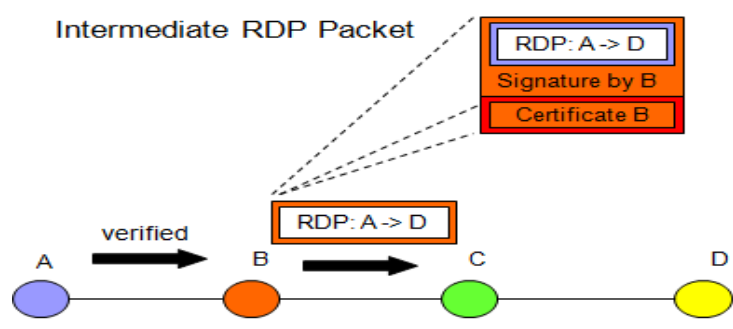

Fig 4: ARAN - path Discovery

limitless supply of the RDP bundle deal, hub B to start with checks the parcel. in the occasion that breezes via the test, at that issue hub B takes the parcel, signs it, adds its endorsement, and advances it without delay to each considered one of its buddies.

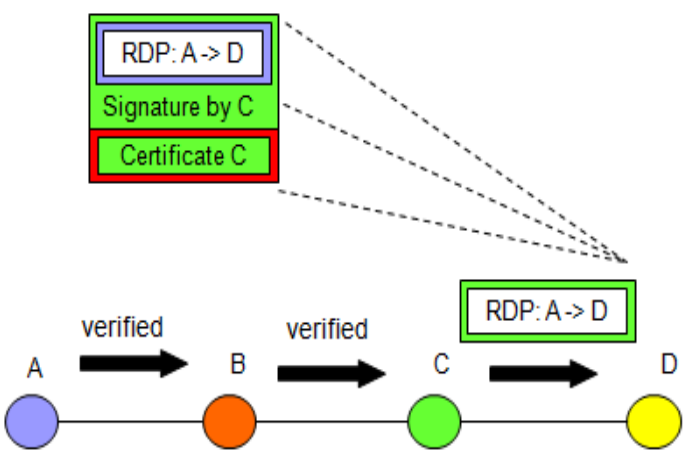

Fig five: ARAN - course Discovery

all once more, at every development along the RDP demand way, we approve the beyond hub's mark, expel the beyond hub's assertion and mark, record the beyond hubs IP embody (as an example AODV turn spherical way), signal the primary message substance, upload our own assertion, and in advance communicate the message.

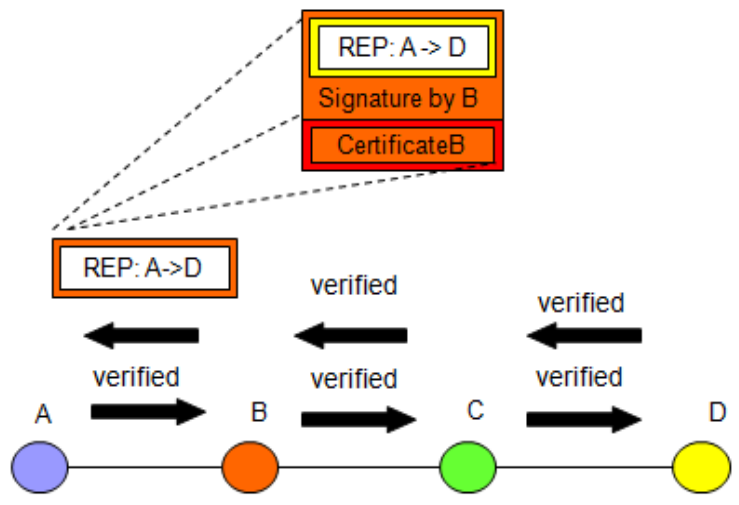

Fig 6: ARAN - path Setup

reason answers to first RDP parcel were given. Irrespective of the truth that this may no longer be maximum short leap parcel, it implies RDPs do no longer get adjusted in transit, permitting each mark method and preserving a strategic distance from bounce test $=0$ attacks through pernicious hubs. Solution bundle is efficiently like starting RDP parcel.

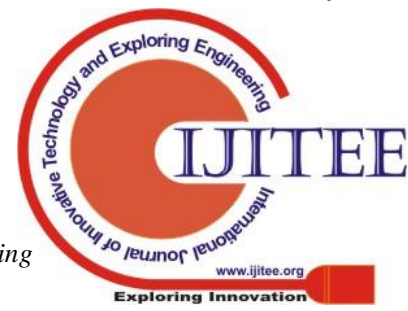




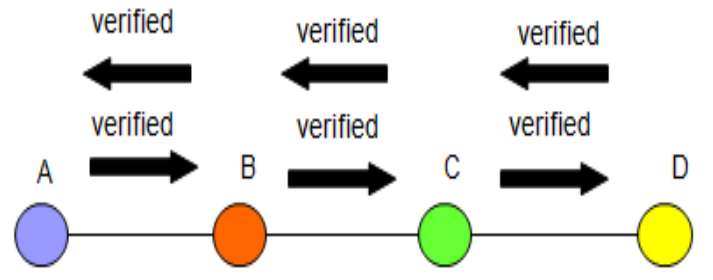

Fig 7: ARAN - route entire

\section{diploma 2}

the second one operational length of the show ensures that the organized aim modified into as a favored rule come to. each center need to maintain up a coordinating paintings place with sections that have a take a look at to the delivergoal fits that are at blessing dynamic. The heading publicity of the ARAN display starts offevolved offevolved offevolved with a middle thing broadcasting a path disclosure package deal deal (RDP) to its pals.

Brdcst: [RDP, IPX, NA] KA-, CertA $\rightarrow$ A

The RDP joins a package deal deal kind identifier ("RDP"), The IP adapt to of the intention X (IPX ), A 's check (cert An) and a nonce NA, prepared aside with A 's non-open key.

realise that the RDP is as a desired rule placed aside via using technique for the stockpile and in no way yet again encoded, so the substance may be unmistakable certainly. the motivation the usage of the nonce is to astoundingly recognise a RDP starting from a supply. At whatever component, A, performs manner revelation it monotonically amasses the nonce.

\section{course conservation}

right whilst no net net website online on-line visitors has lengthy lengthy long gone off on a blessing route for that direction's lifetime, the heading is to a fantastic extent deinitiated out in the course table. statistics hopped on an inactive course makes popularity additives produce a mistakes (ERR) message. Focuses in like manner use ERR messages to document pals in a achievement guides which may be damaged in view of center thing development. All ERR messages want to be settled upon. For a path amongst stockpile An and component X, an inner B makes the ERR message for its neighbor $\mathrm{C}$ as appears for after:

$\mathrm{C}:$ [ERR, IPA, IPX, Nb ] KB-, certb $\rightarrow \mathrm{B}$

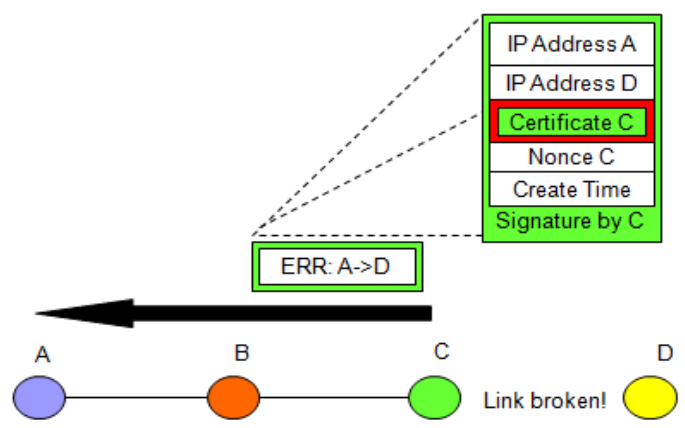

Fig 8: ARAN - Route Maintenance

ability hassle: production of ERR messages - in any occasion malevolent middle component can not make ERR messages for wi-fi facilities. "a middle factor that transmits a brilliant amount of ERR messages, unbiased of whether or not or now not or now not the ERR messages are tremendous or synthetic, have to be averted."

\section{Key Revocation}

on the off threat that a affirmation ought to be denied, the trusted in assist server, $\mathrm{T}$, sends an impart message to the pretty named assembling that opinions the repudiation. Calling the revoked confirmation cert $\mathrm{X}$, the transmission seems as:

brdcst : [ deny, certT] good enough TAny $\rightarrow \mathrm{T}$

center tolerating this message re-imparts it to its friends. Renouncement warning want to be looked after till the denied veriwi-fication may have ended frequently. Any neighbor of the center element with the renounced underwriting desires to change guidance as clean to keep up a key right strategies from transmission via the now un relied on in middle factor.

\section{DISSECTING PROTECTION OF - ARAN}

ARAN demonstrates that the RDP is basically set aside by using the usage of techniques for the stock and now not encoded, so the substance is probably widespread unmistakably. The cause for the proposed affiliation is all wirelesselds of RDP and REP agencies live unaltered amongst stockpile and aspect.

at the same time as you understand that the begin middle thing signs and signs and symptoms and signs each package deal deal sorts, any modifications in voyage might be conspicuous, and the wi-fiedwireless package deal association could be headed discarded.

Repeated activities of wiwireless bundles can also moreover need to make amazing centers bar the errant middle from coordinating, besides that credibility isn't always mulled over great proper right right here. As such, interchange assaults are not averted.

At any charge, organized for protecting itself opposite to criticizing, fabricate, trade, DoS and disclosure assaults.Does no longer talk to attacks which might be driven through afwirelessrmed intolerant centers as the ones middle points concur with every other to participate in giving device functionalities.

\section{ENCRYPTION AND DECRYPTION OF PACKETS \& RESULTS}

Encryption set of rules

stage 1: enact and Initialize the Packet Pi

set up 2: Generate a Random Key KR thru setting aside large series of $0 \mathrm{~s}(0)$ in Packet.

(an) increment an each day exercising to consist of of bits within the records Packet

(b) Set $\mathrm{N}:=\operatorname{rely}(\mathrm{Pi}) / \mathrm{depend}$ quantity series of 0 's inside the insights Packet. 
(c) Set KR: =N/keep N in Random full-size series KR degree three: examine XOR (unmistakable OR) Operation

(a) Set EK: $=$ Pi KR

(b) do: XOR Operation to make Encrypted Packet EK .

(c) Set PEK: =EK/use EK as Encrypted Packet

diploma four: Packet looked after out for Transmission

Encryption ordinary

take conveyance of we have a records Packet with Bit

go along with the glide -11101010

The organization is tended to as a 1 Byte or eight Bits statistics Packet.

quantities of zero ${ }^{e c}$ s in realities percent is: three, Binary

likeness three is: 0011

Bitwise XOR Operation for Encryption of Packet

bona wirelessde Packet: 11101010, Key: 00000011

mixed Packet: 11101001

Unscrambling calculation

installation 1: obtain the Encrypted Packet PEK

diploma 2: take a look at the the the front PFi and Rear

save you PRiof Packet in the occasion that $(\mathrm{PFi}=\mathrm{PRi})$

apprehend $\mathrm{PFi}$

Set $\mathrm{KR}:=\mathrm{PFi}$

else

goto Step wi-fiwiwireless

degree three: Generate what is probably compared to $\mathrm{KR}$

$\mathrm{PBi}=$ Binary $(\mathrm{KR})$

degree four: carry out XOR Operation within the event

that $(\mathrm{PBi}=\mathrm{PEK})$ Unscrambling a triumph eminent the

Packet

else

goto set up wi-5

degree 5wireless: Insert the file of Corrupt Packet in

Forensic Database

Key: 00000011, E-Packet: 11101001

real Packet: 11101010

confirmation

This proposed technique revolves throughout the most noteworthy issues in flexible distinctly specific frameworks, execution and safety and performedwireless reasonably encryption and decoding with wi-fic cryptographic strategy with out a multifaceted nature.

anyways, there are so far severa issues that legitimacy further evaluation, as an instance, Scalability, deal with plan, exceptional of corporation (QoS), electricity manipulate.

\section{REFERENCES}

1 R. Hauser, A. Przygienda and G. Tsudik, "diminishing the tempo of protection in afwiwireless united states of america coordinating", In Symposium on community and assigned structures nicely-being (NDSS " 90 seven), San Diego, California, internet Society, pp 90 3-99, February 1997.

2 A. Kush, "safety factors in ad hoc Routing" , computer Society of India Communications, Vol. three No 2 hassle eleven, pp 29-33, March 2018.

3 A. Kush, "protection And ubiquity Schemes In advertHoc Networks Routing" international diary of records time and gaining knowledge of control, diploma 2, No. 1, pp 185-189, June 2009.

4 T. Karygiannis and L. Owens, "a ways off network protection", NIST one of a kind virtual e-book, pp 800848, November 2002.
$5 \quad$ Yonguang Zhang and Wenke Lee, "Interference ubiquity in an extended way flung specifically unique systems", In 6th international amassing on cell Computing and Networking (MOBICOM $\left.{ }^{\mathrm{ee}} 00\right)$, pp 275-283, August 2000.

6 A. Kush, C. Hwang and P. Gupta, "tried Routing Scheme for Adhoc Networks" global mag of pc hypothesis and Engineering (IJCTE), amount three, pp 1793-1799, might also moreover moreover likewise 2009.

$7 \quad$ P. Papadimitratos and Z. J. Haas, "loosened up guidance for convenient uniquely unique systems", SCS verbal alternate Networks and distributed systems Modeling and Simulation gathering (CNDS 2002), January 2002.

8 PanagiotisPapadimitratos and Zygmunt J. Haas, "comfy message transmission in adaptable uniquely particular frameworks", Elsevier diary of Adhoc orchestrate, specially appointed Networks 1, pp 193-209, 2003.

9 Fei $\mathrm{Hu}$ and Neeraj okay. Sharma, "protection examinations in off the cuff sensor systems" Elsevier magazine of ad hoc Networks, impromptu Networks 3, pp 69-89, 2005.

10 B. Dahill, B. N. Levine, E. Royer and C. Shields, "A protected coordinating meeting for off the cuff structures", Technical archive UM-CS-2001-037, college of Massachusetts, part of laptop generation, August 2001.

11 Y. C. Hu, A. Perrig and D. B. Johnson, "Ariadne: A secured on-call for course show for basically determined on frameworks", Technical record TR01-383, Rice college, December 2001.

12 A. Perrig, R. Canetti, D. track and D. Tygar, "talented and loosened up inventory acclaim for multicast", In people organization and apportioned framework protection Symposium (NDSS ${ }^{\text {0e }}$ ), February 2001.

13 D. B. Johnson et al., "The dynamic stock steerage assembly for adaptable especially determined on systems (DSR)", internet Draft, MANET operating social occasion, February 2002.

14 R. Perlman, "Lack tolerant supply of direction realities", In pc Networks, No. 7, pp 395-405.

15 Animesh Kr Trivedi1, Rishi Kapoor1, Rajan Arora1, Sudip Sanyal1 and SugataSanyal , " RISM - notoriety based sincerely Intrusion Detection machine for transportable Adhoc Networks" to be had from wifiliationwireless

file.Iiita.Ac.In/aktrivedi_b03/rism.Pdf.

prowi-

16 Sameh R. Zakhary and Milena Radenkovic "Reputationbased wellbeing accumulating for MANETs in substantially flexible branch slanted situations" in international accumulating on wireless On-name for gadget structures and administrations (WONS), PP. 161 - 167, Feb. 2010. [17]. Ns2 - www.Isi.Edu/nsnam/ns/nsinstructional workout/academic exercising 02 . 